ARTICLE

https://doi.org/10.1038/s41467-020-19510-5

\title{
Organized cannabinoid receptor distribution in neurons revealed by super-resolution fluorescence imaging
}

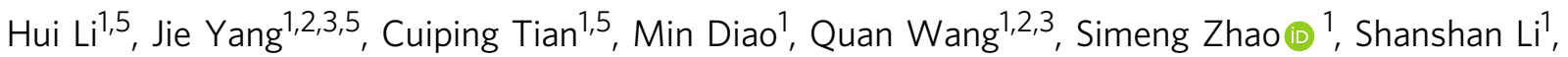
Fangzhi Tan (1) 1, Tian Hua (1) 1, Ya Qin ${ }^{3}$, Chao-Po Lin' ${ }^{3}$, Dylan Deska-Gauthier ${ }^{4}$, Garth J. Thompson (1) 1, Ying Zhang ${ }^{4}$, Wenqing Shui ${ }^{1,3}$, Zhi-Jie Liu (1) ${ }^{1,3}$, Tong Wang ${ }^{3}$ \& Guisheng Zhong (1) ${ }^{1,3 凶}$

G-protein-coupled receptors (GPCRs) play important roles in cellular functions. However, their intracellular organization is largely unknown. Through investigation of the cannabinoid receptor $1\left(C_{1}\right)$, we discovered periodically repeating clusters of $C B_{1}$ hotspots within the axons of neurons. We observed these $\mathrm{CB}_{1}$ hotspots interact with the membrane-associated periodic skeleton (MPS) forming a complex crucial in the regulation of $\mathrm{CB}_{1}$ signaling. Furthermore, we found that $\mathrm{CB}_{1}$ hotspot periodicity increased upon $\mathrm{CB}_{1}$ agonist application, and these activated $C_{1}$ displayed less dynamic movement compared to non-activated $C_{1}$. Our results suggest that $C B_{1}$ forms periodic hotspots organized by the MPS as a mechanism to increase signaling efficacy upon activation.

\footnotetext{
${ }^{1}$ iHuman Institute, ShanghaiTech University, 201210 Shanghai, China. ${ }^{2}$ University of the Chinese Academy of Sciences, 100049 Beijing, China. ${ }^{3}$ School of Life Science and Technology, ShanghaiTech University, 201210 Shanghai, China. ${ }^{4}$ Department of Medical Neuroscience, Dalhousie University, Halifax, NS

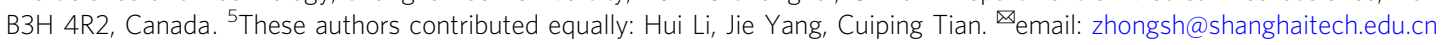


G -protein-coupled receptors (GPCRs) are a large family of membrane proteins that play important roles in cellular functions by initializing a variety of intracellular processes via neurotransmitters and hormone signaling. To fulfill their function, GPCRs are likely anchored by cellular skeletal structures facilitating their interactions with intracellular protein complexes. Therefore, their membrane organization and relationship with skeleton proteins, which are still largely unknown, is likely critical for their cellular function. In addition, it would be important to know if diverse GPCRs share similar structural organizations.

Most GPCRs are expressed at low levels in native tissue. Typically, investigators must overexpress them in cultured cells in order to study their structures and functions. The cannabinoid receptor $1\left(\mathrm{CB}_{1}\right)$ is one of the highest-expressed GPCRs in the central nervous system, making it feasible to study in its natural state $^{1}$.

$\mathrm{CB}_{1}$ is important for many biological functions such as pain, mood and memory ${ }^{2-5}$. Recent structural studies have revealed the isolated atomic arrangement of $\mathrm{CB}_{1}{ }^{6,7}$. However, these studies have primarily used X-ray crystallography or cryo-electron microscopy techniques that require high concentrations of wellpurified proteins in detergent solution. $\mathrm{CB}_{1}$ structure has rarely been studied within cells in combination with signaling proteins, integral membrane proteins, or other membrane-associated proteins. Thus, the $\mathrm{CB}_{1}$ cellular structure remains incomplete, limiting our ability to fully exploit its function.

Super-resolution microscopy, an imaging method that overcomes the diffraction limit of conventional microscopy, has led to the discovery of the membrane-associated periodic skeleton (MPS) in the axons of neurons ${ }^{8}$. This highly ordered submembrane skeletal structure can play many roles in neuronal function, including acting as a flexible mechanical support, organizing membrane protein distribution, and the development of axons and dendrites. The discovery of the MPS, and the many studies that have characterized cellular structures across different cell types ${ }^{9,10}$, demonstrate the power of super-resolution imaging for uncovering intracellular structures at the nano-scale. Recently, using a type of super-resolution imaging called STORM, Zhou et al. ${ }^{11}$ proposed a model where $\mathrm{CB}_{1}$ forms a periodic pattern when activated. They found $\mathrm{a} \sim 190 \mathrm{~nm}$ periodic pattern of $\mathrm{CB}_{1}$ in cultured hippocampal neurons exclusively under the administration of agonists. A previous study, using a similar imaging technology, also revealed distinct $\mathrm{CB}_{1}$ structures across different cell types in the brain, but showed no sign of a periodic pattern ${ }^{12}$. Thus, the intracellular organization of $\mathrm{CB}_{1}$ in neurons remains unclear.

Herein, by employing another type of super-resolution imaging, called stimulated emission depletion (STED), we systematically investigated the nano-structure of $\mathrm{CB}_{1}$ and other GPCRs in brain tissues and primary cultured neurons. Our results revealed a periodic structure of $\mathrm{CB}_{1}$ clusters along the axons of inhibitory interneurons. These $\mathrm{CB}_{1}$ clusters were organized into "hotspots" $\sim 190 \mathrm{~nm}$ apart. Using dual-color STED imaging and cellular biology techniques, we demonstrated that the $\mathrm{CB}_{1}$ hotspots were associated with the MPS. The $\mathrm{CB}_{1}$ hotspots exhibited confined dynamics, which were reduced by receptor activation. Thus, our current studies demonstrate that the $\sim 190 \mathrm{~nm}$ periodic structure of the cytoskeleton appears to be the backbone for intracellular signaling to occur.

\section{Results}

$\mathrm{CB}_{1}$ exhibits semi-periodic hotspots in neurons. To study the structure of $\mathrm{CB}_{1}$ in vivo, we first demonstrated the specificity of our $\mathrm{CB}_{1}$ antibody labeling (Supplementary Fig. 1a, b). We found, in agreement with previous findings, that $\mathrm{CB}_{1}$ is mainly distributed in the axons of inhibitory interneurons, especially in those of cholecystokinin (CCK)-positive inhibitory interneurons (Supplementary Fig. $1 \mathrm{c}, \mathrm{d})^{12} \cdot \mathrm{CB}_{1}$ did express much lower in myelinated axons and in the axons of excitatory neurons (Supplementary Fig. 1e, f).

While we confirmed that $\mathrm{CB}_{1}$ was distributed in the axon shaft, the nature of its distribution was unknown. STED imaging is known to be well suited for studying the nano-scale structure of cellular components in fixed preparations ${ }^{9,13}$. Therefore, we undertook STED imaging, with a spatial resolution around $70 \mathrm{~nm}$ (Supplementary Fig. 2a), of immunolabeled hippocampal tissues to examine $\mathrm{CB}_{1}$ organizations along the axon shaft. $\mathrm{CB}_{1}$ displayed hotspots both with and without apparent periodicity within axons of the same axonal segment (Fig. la-c). We quantified the degree of periodicity using one-dimensional (1D) autocorrelation analysis by projecting the signals to the longitudinal axis of the axon and calculating the average 1D autocorrelation function over many axon segments 8,14 . The 1D autocorrelation amplitude, defined as the average amplitude of the peaks at $\sim 190 \mathrm{~nm}$, quantifies the degree of periodicity of the $\mathrm{CB}_{1}$ hotspots ${ }^{8,14}$.

To identify whether $\mathrm{CB}_{1}$ possess a similar distribution in other brain regions, we performed STED imaging in the cortex, and observed a similar semi-periodicity in neuron axons (Fig. 1d-f). The distance between these rhythmic hotspots was also $\sim 190 \mathrm{~nm}$ in the cortex as it was hippocampus (Fig. 1g). Next, we imaged the structure of $\mathrm{CB}_{1}$ from cultured hippocampal neurons. We found that antibody-labeled $\mathrm{CB}_{1}$ exhibited hotspots with both high and low degree of periodicity in axons of cultured neurons (Supplementary Fig. $2 \mathrm{~b}-\mathrm{d}$ ). These results are comparable to the in vivo results above (Fig. 1a-c).

To avoid artifacts caused by fixation procedures, we performed live super-resolution imaging on cultured neurons. To this end, we used structured illumination microscopy (SIM), a type of super-resolution imaging that is suitable for investigating the live structure of cellular molecules at high spatial resolution ${ }^{15}$. Using SIM microscopy, with a spatial resolution around $122 \mathrm{~nm}$ (Supplementary Fig. 2e), we revealed clusters of $\mathrm{CB}_{1}$ in cultured neurons, and those clusters appeared highly organized as hotspots in some regions of axons (Supplementary Fig. $2 \mathrm{f}-\mathrm{h}$ ). Again, $\mathrm{CB}_{1}$ exhibited both high and low periodic hotspots (Supplementary Fig. $2 \mathrm{f}-\mathrm{h}$ ). The spatial distance between periodic hotspots was $\sim 190 \mathrm{~nm}$, which was comparable to our results with antibody labeling in fixed preparations (Fig. 1g). Notably, the regularity of the $\mathrm{CB}_{1}$ structure in cultured neurons was higher than that in the brain (Fig. 1h). Therefore, we conclude that there is a commonality of the semi-periodic feature of $\mathrm{CB}_{1}$ in neurons, an unexpected characteristic of GPCRs in the cellular membrane.

$\mathrm{CB}_{1}$ is associated with MPS in neurons. The semi-periodic organization of $\mathrm{CB}_{1}$ in axons raised two important questions, how is the $\mathrm{CB}_{1}$ semi-periodic organization formed and what are the complexes related to the semi-periodic organization of $\mathrm{CB}_{1}$ ? To answer the first question, we need to identify the components associated with the semi-periodic complex of $\mathrm{CB}_{1}$ in native states. To this end, we performed mass spectrometry (MS) experiments with modifications to increase the enrichment of membrane proteins in six different regions of the mouse brain (Supplementary Fig. 3). We found that $\mathrm{CB}_{1}$ was expressed at different levels in the six brain regions and that the expression level paralleled the AMPA specific glutamate receptor 1 and $N$-methyl-Daspartate (NMDA) receptor (Supplementary Fig. 3a), which are known to crosstalk with $\mathrm{CB}_{1}$ in the central nervous system ${ }^{16,17}$. Furthermore, we observed the association of GPCR-related signaling molecules with $\mathrm{CB}_{1}$ (such as $\mathrm{Gi}$, tyrosine-protein kinase 
a
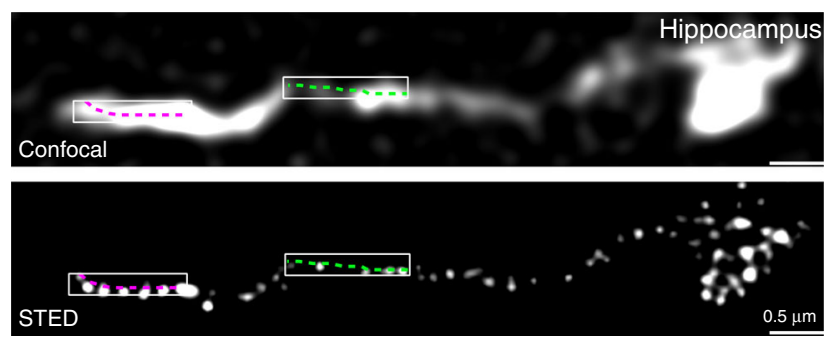

b

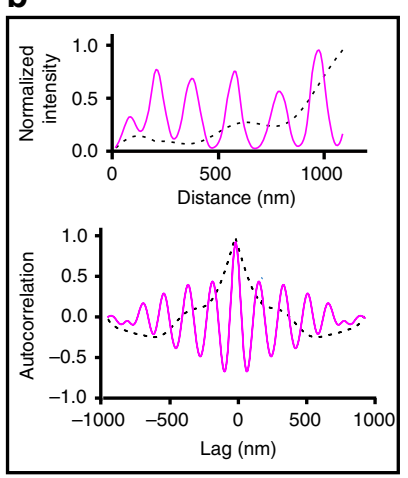

C

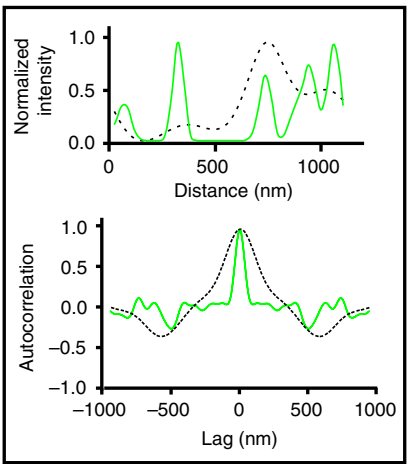

g

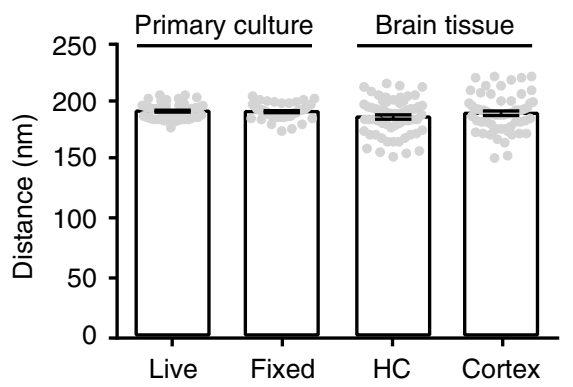

d
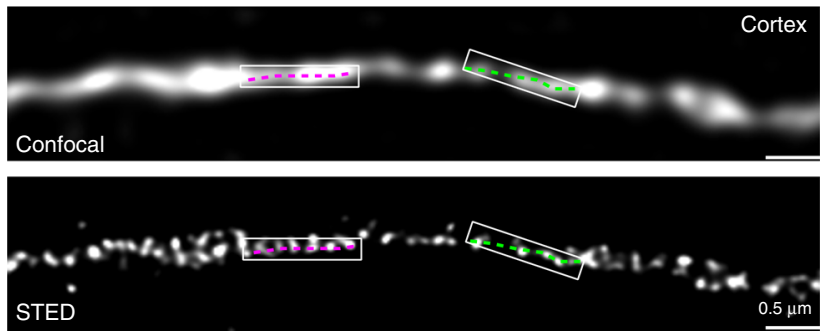

e

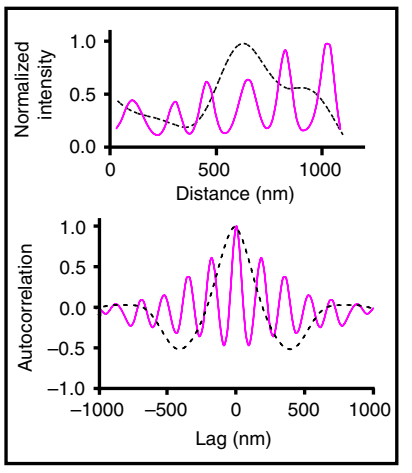

f

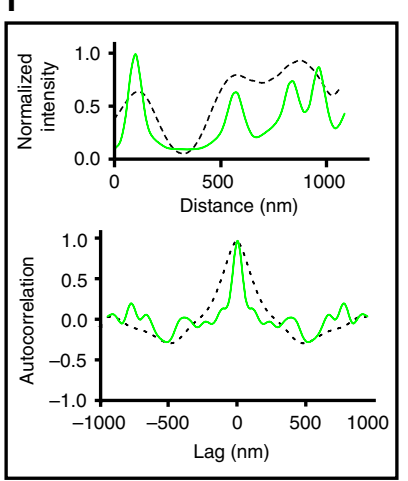

h

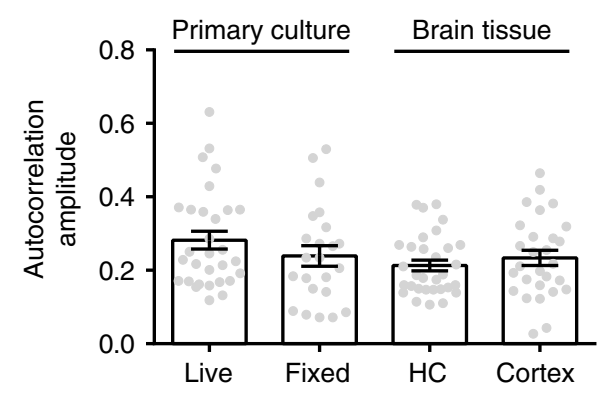

Fig. 1 Periodic hotspots of $\mathbf{C B}_{\mathbf{1}}$ of varying strength in axons from brain tissue. a Representative confocal and corresponding $S T E D$ images of $C B_{1}$ in the hippocampus of mature $\mathrm{C} 57$ mice. $N=3$ biological replicates. $\mathbf{b}, \mathbf{c}$ Intensity plotted along the lines in the box regions (top graph). Autocorrelation analysis for the confocal and STED images (bottom graph). d-f Similar to (a-c), but in the cortex region of mature C57 mice. $N=3$ biological replicates. $\mathbf{g}$ Histogram of $\mathrm{CB}_{1}$ spacing in different samples, live and fixed hippocampal neurons, brain tissue of the hippocampus (HC) and cortex. Data are mean \pm s.e. $\mathrm{m}$. ( $N=3$ biological replicates; 70-120 axonal regions were examined per condition). $p=0.09$, (no significance), one-way ANOVA. Actual spacing (from left to right), $192 \pm 0.8 \mathrm{~nm}, 192 \pm 0.8 \mathrm{~nm}, 187 \pm 2 \mathrm{~nm}, 190 \pm 2 \mathrm{~nm}$. h Amplitude of the average autocorrelation functions calculated from randomly selected axon segments in different samples. $p=0.13$ (no significance), one-way ANOVA. Actual autocorrelation amplitude (from left to right), $0.28 \pm 0.02,0.24 \pm$ $0.03,0.21 \pm 0.01,0.23 \pm 0.02$. Data in $(\mathbf{g}, \mathbf{h})$ are mean \pm s.e.m. $(N=3$ biological replicates; $70-120$ axonal regions were examined per condition). Source data are provided as a Source Data file.

and Fyn), indicating the reliability of our modified MS method (Supplementary Fig. 3b).

Next, we compared the expression extent of selected MPS components with $\mathrm{CB}_{1}$ expression. While different isoforms of ankyrin expressed across different functional domains of neurons, ankyrin-B (ankB) is predominantly distributed in the axons of neurons ${ }^{14,18}$. Our MS experiments indicated that the expression level of $\mathrm{CB}_{1}$ was highly correlated to that of ankB, but not ankyrin-R (ankR) (Supplementary Fig. 3a). Further, the expression level of $\mathrm{CB}_{1}$ was correlated to that of both aII-spectrin and $\beta$ II-spectrin (Supplementary Fig. 3a) while other membrane proteins seem unrelated to the expression levels of $\mathrm{CB}_{1}$ (Supplementary Fig. 3c).

Earlier studies showed that MPS, like ankyrin, spectrin, displayed a highly coordinated pattern with an interval around $190 \mathrm{~nm}$, which resembles the structure of $\mathrm{CB}_{1}$ in some regions of neurons revealed both by STED and SIM imaging experiments. Thus, consistent with our MS experimental results, there could be a tight association between $\mathrm{CB}_{1}$ and MPS components. To test the above view, we carried out two independent types of experiments, two-color STED imaging and proximity ligation assays (PLA) ${ }^{19}$. First, we visualized the spatial relation of $\mathrm{CB}_{1}$ and MPS molecules by dual-color STED imaging. The MPS was visualized through immunolabelling of the C-terminus of $\beta I I-s p e c t r i n$, which is located at the center of each spectrin tetramer connecting adjacent actin rings ${ }^{14,18}$. We quantified the extent of colocalization between $\mathrm{CB}_{1}$ and $\beta \mathrm{II}$-spectrin using $1 \mathrm{D}$ cross-correlation analysis to calculate the average $1 \mathrm{D}$ cross-correlation function between the two color channels over randomly chosen axon segments ${ }^{8}$. At periodic hotspots, $\mathrm{CB}_{1}$ displayed high colocalization with $\beta$ II-spectrin while at nonperiodic clusters, $\mathrm{CB}_{1}$ displayed little colocalization with $\beta$ II-spectrin (Fig. 2a-c). Previous studies demonstrated that ankB mediated the attachment of membrane proteins to the MPS, and is located in the middle region of each spectrin tetramer ${ }^{18,20}$. Thus, we reasoned that $\mathrm{CB}_{1}$ 's interaction with ankB may organize the semi-periodic 
a
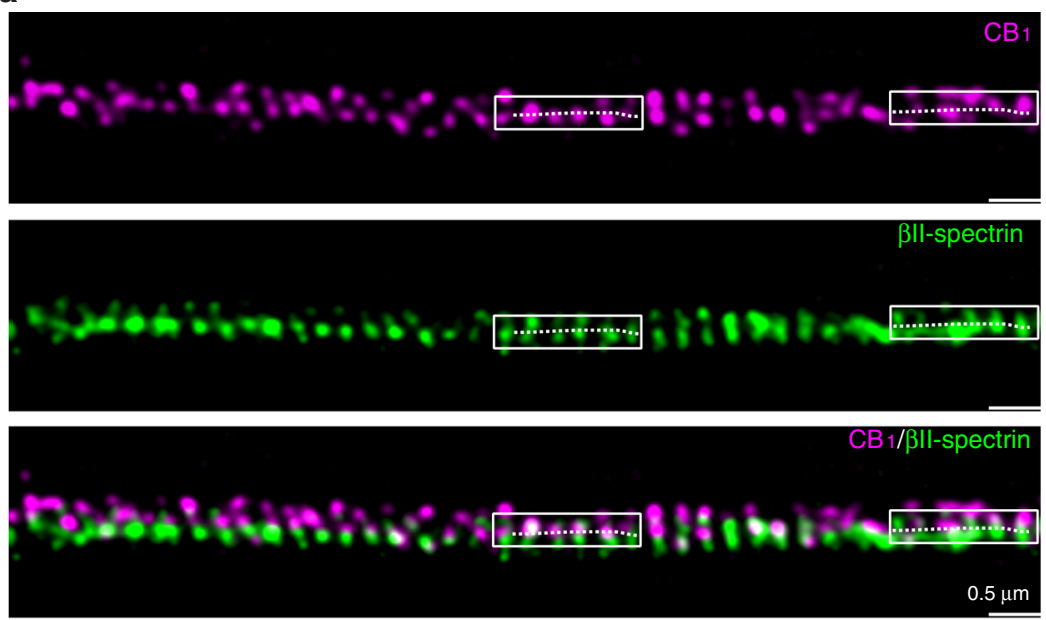

b
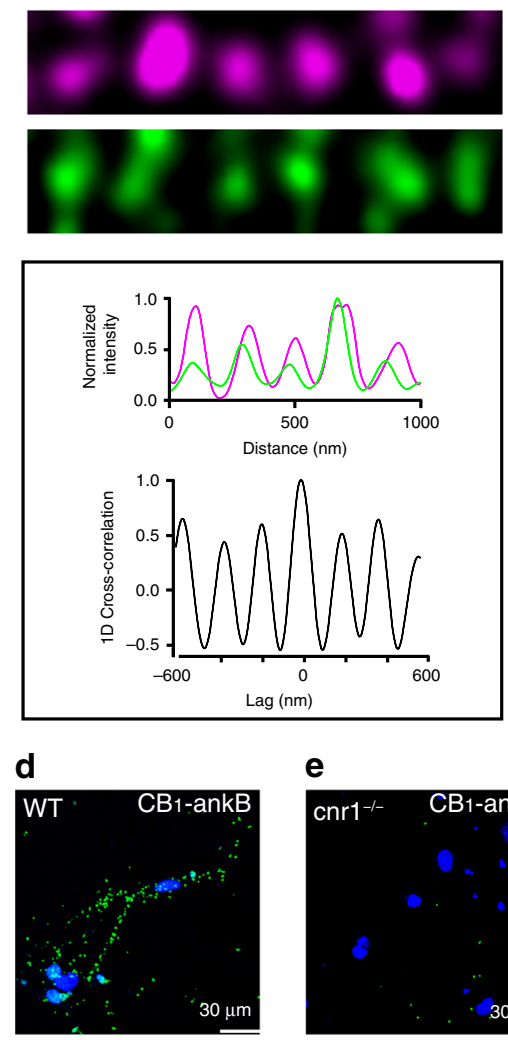

e

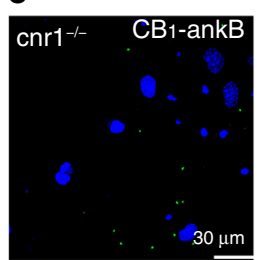

f

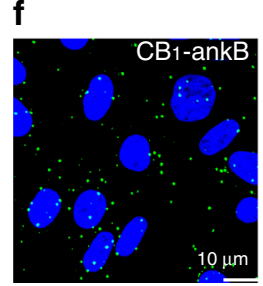

g

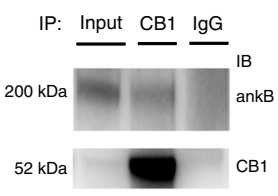

c
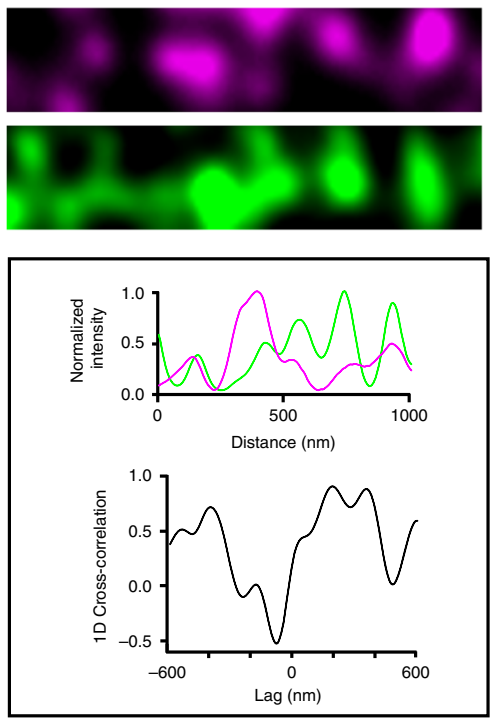

h
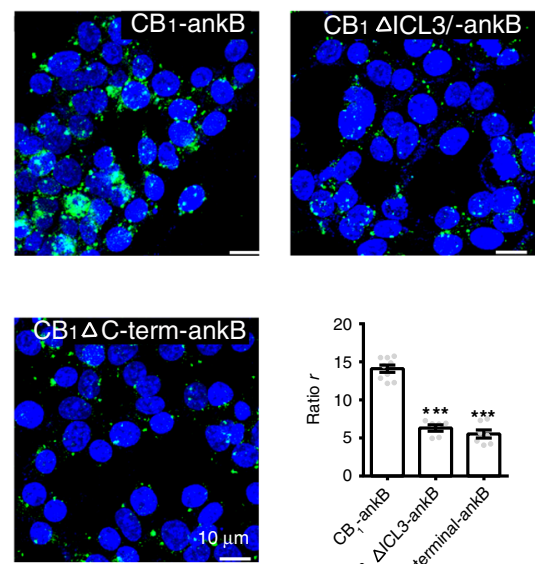

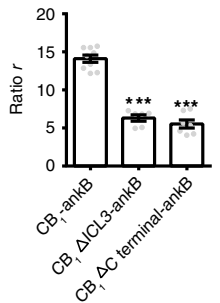

pattern of $\mathrm{CB}_{1}$ with the MPS. Indeed, at periodic hotspots, $\mathrm{CB}_{1}$ displayed high colocalization with ankB while at nonperiodic clusters, $\mathrm{CB}_{1}$ displayed little colocalization with ankB (Supplementary Fig. $4 a-c)$. Furthermore, the spatial distance between periodic $\mathrm{CB}_{1}$ hotspots was comparable to both those of $\beta \mathrm{II}-$ spectrin and ankB (Supplementary Fig. 4d). These results

unambiguously show that $\mathrm{CB}_{1}$ forms a semi-periodic complex associated with the MPS.

Then, to demonstrate the association between $\mathrm{CB}_{1}$ and the complex components identified, we carried out PLA experiments, another type of imaging assay that reliably detects the close physical distribution of two subjects ${ }^{19}$. It is known that $\alpha$-adducin 
Fig. $2 \mathbf{C B}_{\mathbf{1}}$ hotspots are connected with components of the MPS. a Two-color STED images of $\mathrm{CB}_{1}$ (magenta) and $\beta \|$-spectrin (green) in the axons of cultured neurons. $N=3$ biological replicates. b, $\mathbf{c}$ Top, enlarged images taken from white boxes from (a). 1D projection traces of $\beta / l$-spectrin (green) and $\mathrm{CB}_{1}$ (magenta) signals along the axon are shown in the middle. $1 \mathrm{D}$ cross-correlation functions between the distributions of $C B_{1}$ and $\beta \|$-spectrin from $\mathrm{CB}_{1}$-positive axon segments are shown in the bottom. d-f PLA was performed in cultured neurons of WT mouse (d), $\mathrm{cnr} \mathbf{1}^{-/-}$mouse (e), and tetracycline-induced $\mathrm{CB}_{1}$ transfected $\mathrm{CHO}$ cells (f) with antibody of $\mathrm{CB}_{1}$ and ankB. Cell nuclei were stained with DAPI (blue). $N=3$ biological replicates. $\mathbf{g}$ Immunoprecipitation of $\mathrm{CB}_{1}$ and ankB in $\mathrm{CB}_{1}-\mathrm{CHO}$ cells. Samples were processed for immunoprecipitation with either anti-CB or lgG control antibodies. Immunoprecipitates were immunoblotted with the anti- $\mathrm{CB}_{1}$ antibody (55kD) and anti-ankB antibody (220kD). N=2 biological replicates. $\mathbf{h}$ PLA was performed in HEK-293T cells coexpressing ankB and different fragments of $\mathrm{CB}_{1}$, including wild type $\left(\mathrm{CB}_{1}\right), \mathrm{CB}_{1}$ with truncated ICL3 loop $\left(\mathrm{CB}_{1} \Delta \mathrm{ICL} 3\right)$, or $\mathrm{CB}_{1}$ with truncated $C$-terminal $\left(C_{1} \Delta C\right.$-term). ratio $r$ from left to right: $14.1 \pm 0.5,6.3 \pm 0.4,5.5 \pm 0.4$. Data are mean \pm s.e.m. $(N=3$ biological replicates; $25-50$ imaging regions were examined per condition). ${ }^{\star \star} p<0.0001$, statistical analysis was performed by unpaired two-tailed Student's $t$ test. Source data are provided as a Source Data file.

and $\beta$ II-spectrin closely distribute in neurites. Indeed, in $c n r 1^{-1-}$ mouse, PLA signal with $\alpha$-adducin and $\beta$ II-spectrin was not changed (Supplementary Fig. 5a, b), indicating the reliability of PLA to detect proteins within a short distance. Then, we determined whether $\mathrm{CB}_{1}$ localizes closely with MPS components in cultured neurons. PLA signals of $\mathrm{CB}_{1}$ and ankB were observed in cultured primary neurons (Fig. 2d), but no PLA signals were detected in the $\mathrm{cnrl}^{-1-}$ mouse neurons (Fig. 2e). Upon the induction of $\mathrm{CB}_{1}$ in the presence of tetracycline, close physical distances were detected between $\mathrm{CB}_{1}$ and several MPS components, including ankB and $\beta \mathrm{II}$-spectrin in $\mathrm{CB}_{1}-\mathrm{CHO}$ cell lines (Fig. 2f and Supplementary Fig. 5c, d). Together, these results from neurons and inducible $\mathrm{CB}_{1}-\mathrm{CHO}$ cell lines clearly illustrate the close physical distance between $\mathrm{CB}_{1}$ and MPS components.

In order to show that $\mathrm{CB}_{1}$ interacted with ankB in tetracyclineinduced $\mathrm{CB}_{1}-\mathrm{CHO}$ cells, we performed immunoprecipitation experiments (Fig. 2g). To identify which parts of $\mathrm{CB}_{1}$ may mediate the interaction between $\mathrm{CB}_{1}$ and ankB, we truncated the third intracellular loop (ICL3) or C terminal of $\mathrm{CB}_{1}$. Notably, the PLA signals were significantly decreased in both conditions, suggesting that both regions, ICL3 and C-terminal, play a role in the interaction between $\mathrm{CB}_{1}$ and ankB (Fig. $2 \mathrm{~h}$ ), and that neither region alone is sufficient to mediate their interaction. Together, these results support the view that $\mathrm{CB}_{1}$ may form a complex with cytoskeleton-related proteins, such as ankB, $\alpha \mathrm{II}$-spectrin and $\beta \mathrm{II}-$ spectrin.

$\mathrm{CB}_{1}$ structure in different compartments of axons. We examined whether the close relation between $\mathrm{CB}_{1}$ and cytoskeleton components locates in axons or dendrites of neurons. In order to achieve this, we first performed PLA labeling, and then immunolabeled either axons or dendrites with tuj1 or MAP2 antibody, respectively. Our results show that the PLA signal between $\mathrm{CB}_{1}$ and ankB substantially diminished after the second round of immunolabeling. We further explored the PLA signal between $\mathrm{CB}_{1}$ and spectrin, which can survive the immunolabeling procedure. Our results clearly showed that the PLA signal located in axons but not in dendrites (Supplementary Fig. 5e-f), indicating that $\mathrm{CB}_{1}$ is associated with MPS components specifically in axons.

Then, we used specific antibodies to label different regions of axons, such as axon initial segments (AISs) with $\beta I V$-spectrin antibody or presynaptic sites with VGAT antibody. Our results show that select AISs express $\mathrm{CB}_{1}$ with periodic pattern with an interval around $190 \mathrm{~nm}$ (Supplementary Fig. 5g, h, l-n) while some AISs did not express $\mathrm{CB}_{1}$ (Supplementary Fig. 5g, i). Interestingly, presynaptic sites with VGAT labeling did express $\mathrm{CB}_{1}$ (Supplementary Fig. 5j), but most were not periodic (Supplementary Fig. 5k). Thus, our results indicated that $\mathrm{CB}_{1}$ exhibited a specialized pattern in different regions of axons. Given that ankyrin and spectrin are uniformly distributed with a regular patter around $190 \mathrm{~nm}$, there are likely some additional components mediating the $\mathrm{CB}_{1}$ distribution pattern in axons, such as the nonperiodic organization in presynaptic sites.

$\mathrm{CB}_{1}$ displays confined dynamics in neurons. $\mathrm{CB}_{1}$ connects to the MPS through ankB forming semi-periodic hotspots. As such, we would expect $\mathrm{CB}_{1}$ to display confined dynamics by interacting with the MPS through ankB. We investigated the dynamics of individual hotspots of $\mathrm{CB}_{1}$ with live SIM imaging. Neurons were ectopically expressed with a $\mathrm{CB}_{1}$-RFP fusion protein (RFP protein is fused to the end of $\mathrm{CB}_{1} \mathrm{C}$-terminal) and live SIM images were acquired 1 day after transfection. In order to identify whether $\mathrm{CB}_{1}$-RFP was recruited to the native site, we performed two-color STED imaging of $\mathrm{CB}_{1}$-RFP and $\beta \mathrm{II}$-spectrin. $\mathrm{CB}_{1}$-RFP displayed highly periodic hotspots that colocalized with $\beta I I-$ spectrin, as well as nonperiodic clusters that did not colocalize with $\beta I I-s p e c t r i n$ (Supplementary Fig. $6 \mathrm{a}-\mathrm{c}$ ) These results were similar to the spatial distribution between $\mathrm{CB}_{1}$ and $\beta \mathrm{II}$-spectrin (Fig. 2a-c), supporting that $C_{1}-R F P$ is transported to $C_{1}$ native sites. We proceeded to examine the dynamics of $\mathrm{CB}_{1}$-RFP using live SIM imaging. Periodic $\mathrm{CB}_{1}$ hotspots were confined to movements around their starting positions displaying confined displacement changes (average $69 \mathrm{~nm}$ ) between time frames (Fig. 3a-c). Averaged autocorrelation analysis of $\mathrm{CB}_{1}$ at different time frames showed a similar periodic distribution, indicating that $\mathrm{CB}_{1}$ clusters maintained their periodicity through time (Fig. 3d). The cross-correlation of neighboring time points was calculated showing that the average cross-correlation value peaked at zero point (Fig. 3e), indicating little or no systematic shift of the $\mathrm{CB}_{1}$ periodicity across different time frames. The periodic wavelength was around $190 \mathrm{~nm}$ at different time frames (Fig. 3f). We compiled the moving traces of individual hotspots of $\mathrm{CB}_{1}$ that clearly indicated confined movement both at short ( $2 \mathrm{~min}$ ) (Fig. $3 \mathrm{~g}$ ) and longer (6 min) (Supplementary Fig. $7 \mathrm{a}-\mathrm{g}$ ) imaging time frames. The moving traces between the two time frames were comparable (Supplementary Fig. $7 \mathrm{~h}$ ), suggesting that $\mathrm{CB}_{1}$ displayed a confined movement around its anchoring point, likely mediated by ankB.

Active $\mathrm{CB}_{1}$ associate with MPS and display less dynamics. $\mathrm{CB}_{1}$ were displayed with coordinated clusters with an interval of around $190 \mathrm{~nm}$ upon WIN application with STED imaging the immunolabeled $\mathrm{CB}_{1}$ in the primary hippocampal neurons (Fig. 4a). Next, we imaged $\mathrm{CB}_{1}$ in the presence of antagonist to test whether blocking $\mathrm{CB}_{1}$ activity had an impact on its organization. Surprisingly, our results show that $\mathrm{CB}_{1}$ still exhibited a periodic pattern in some axons with $\mathrm{CB}_{1}$ antagonist, suggesting that the basal level activation of $\mathrm{CB}_{1}$ might not be necessary for the periodic organization (Fig. $4 \mathrm{a}-\mathrm{c}$ ). All raw STED images were included in Supplementary Fig. 8. To test the effect of $\mathrm{CB}_{1}$ activation on its dynamics, we imaged the dynamics of $\mathrm{CB}_{1}$ in the primary neurons transfected with $C_{1}$-RFP in the presence of 
a

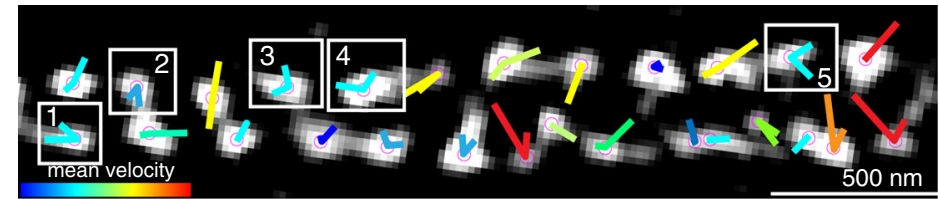

b
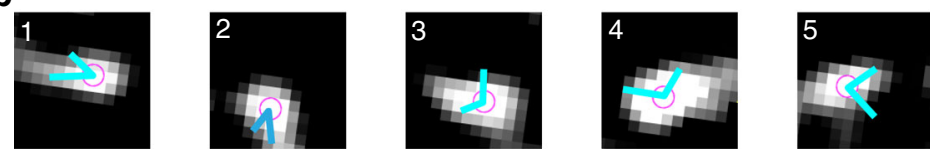

C

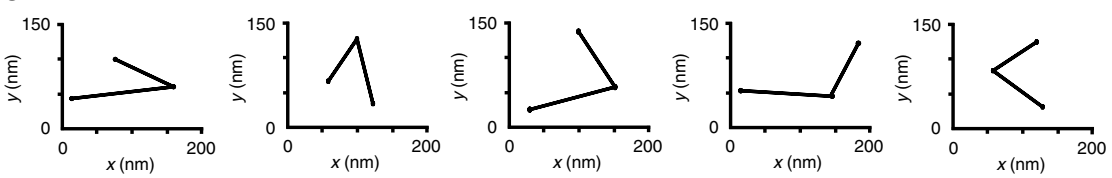

d

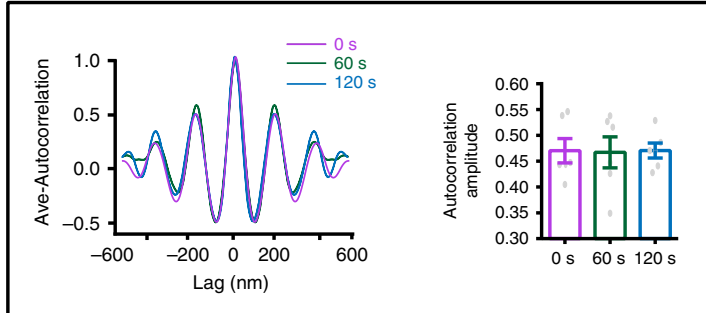

e

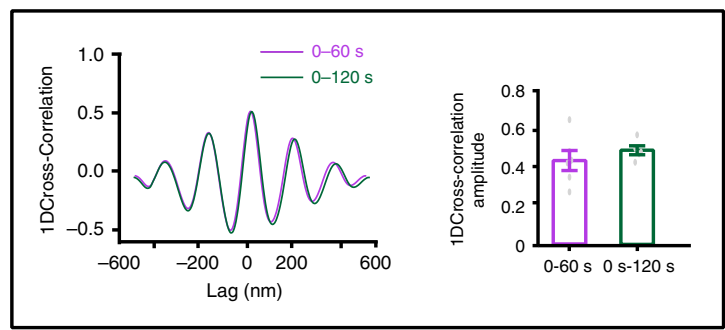

f

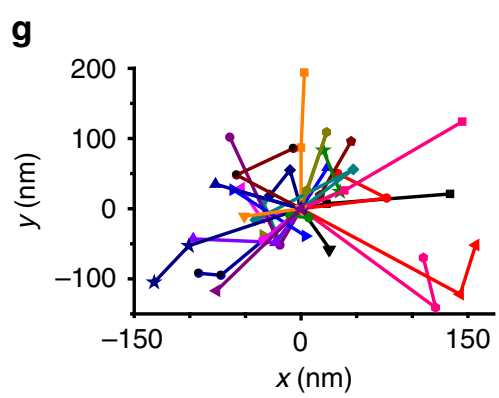

Fig. 3 Periodic $\mathbf{C B}_{\mathbf{1}}$ hotspots display stable dynamics revealed by live $\mathbf{S I M}$ imaging. a Representative live image of transfected $\mathrm{CB}_{1}$-RFP in the primary neuron (DIV 9-12) of the SD rat acquired by SIM. Individual $\mathrm{CB}_{1}$ hotspots are marked with purple balls, and locations of each individual time points are connected with lines. Line colors indicate trace indexes. $N=3$ biological replicates. $\mathbf{b}$ Five $\mathrm{CB}_{1}$ hotspots shown in (a) with their relative locations. c Displacement changes from (b) are around $60-70 \mathrm{~nm}$ between neighboring time points. d Averaged autocorrelation analysis of $\mathrm{CB}_{1}$ distributions at different time points with the histogram of the autocorrelation amplitude. $p=0.99$ (no significance), one-way ANOVA. Actual autocorrelation from left to right, $0.47 \pm 0.02,0.47 \pm 0.03,0.47 \pm 0.02$. e Averaged cross-correlation analysis between the neighboring frames ( 0 vs $60 \mathrm{~s}, 0$ vs $120 \mathrm{~s})$ showed similar distribution properties and histogram showing amplitude of average cross-correlation. $p=0.386$ (no significance), statistical analysis was performed by unpaired two-tailed Student's $t$ test. Actual cross-correlation amplitude (from left to right), $0.45 \pm 0.05 ; 0.51 \pm 0.02$. f The histogram of $\mathrm{CB}_{1}$ spacing across time points. $p=0.69$ (no significance, one-way ANOVA). Actual spacing (from left to right), $192 \pm 0.6,192 \pm 0.7,191 \pm 0.6 \mathrm{~nm}$. Data in (d-f) are mean $\pm \mathrm{s}$. e.m. ( $N=3$ biological replicates; $70-120$ axonal regions were examined per condition). $\mathbf{g}$ Traces of the individual $\mathrm{CB}_{1}$ hotspots over time. Source data are provided as a Source Data file. 
a

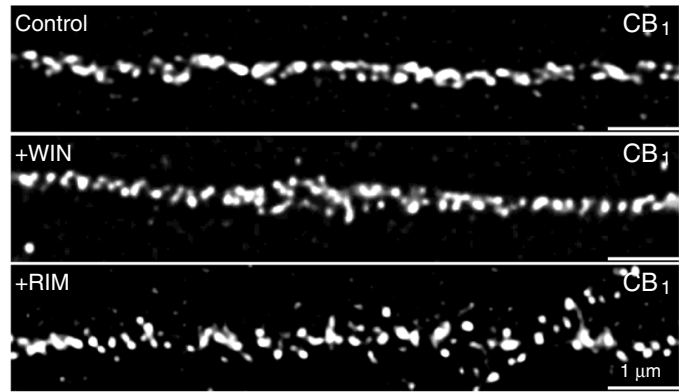

d
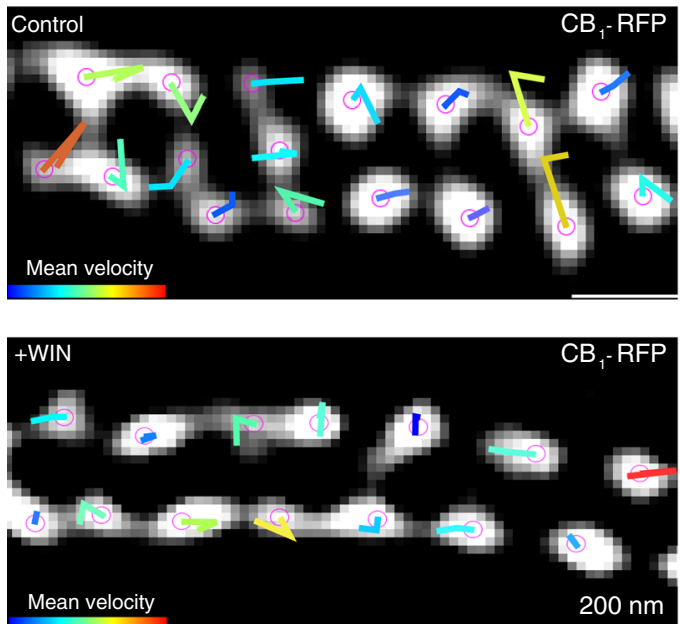

b

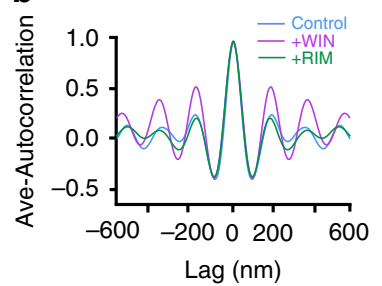

e

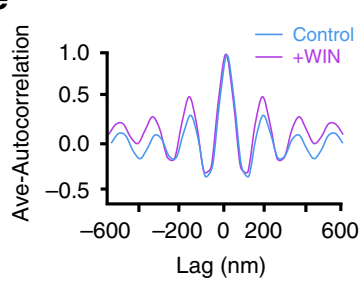

g

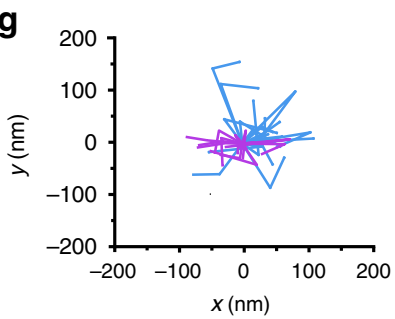

C

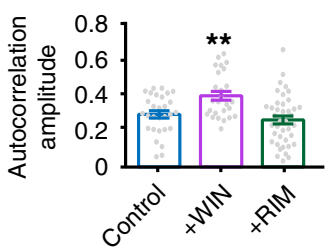

f

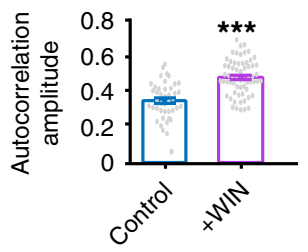

h

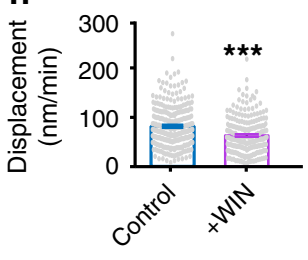

Fig. 4 The dynamics of active $\mathbf{C B}_{\mathbf{1}}$ hotspots. a Representative STED images of $\mathrm{CB}_{1}$ in primary hippocampal neurons for control, with WIN treatment (500 $\mathrm{nM}, 10 \mathrm{~min})$ and RIM $(1 \mu \mathrm{M}, 10 \mathrm{~min})$ treatment. $N=3$ biological replicates. $\mathbf{b}$ Average autocorrelation analysis for the STED images of $\mathrm{CB}_{1}$ in different conditions. $\mathbf{c}$ The histogram of autocorrelation amplitude of $\mathrm{CB}_{1}$ (from left to right), $0.28 \pm 0.02,0.38 \pm 0.02,0.25 \pm 0.02$. $p=0.0003$, ${ }^{\star \star} p<0.01$, one-way ANOVA. d Representative live image of transfected $\mathrm{CB}_{1}$-RFP in untreated neurons (top, "Control"), and neurons treated with WIN (bottom, "+WIN") acquired by SIM. Individual $\mathrm{CB}_{1}$ hotspots are marked with purple balls, and locations of each individual time points are connected with lines. Line colors indicate trace indexes. $N=3$ biological replicates. e Averaged autocorrelation analysis of $\mathrm{CB}_{1}$ distributions in live neurons. $\mathbf{f}$ The histogram of autocorrelation amplitude of $\mathrm{CB}_{1}$ without and with WIN treatment was shown. ${ }^{\star \star \star} p<0.0001$, statistical analysis was performed by unpaired two-tailed Student's $t$ test. Actual autocorrelation amplitude (from left to right), $0.33 \pm 0.02,0.46 \pm 0.01$. $\mathbf{g}$ Traces the dynamics of $\mathrm{CB}_{1}$ hotspots without WIN treatment (magenta) and $\mathrm{CB}_{1}$ hotspots with WIN treatment (blue) over time. $\mathbf{h}$ The displacement of $\mathrm{CB}_{1}$ hotspots in different conditions. Control, $83.9 \pm 2.5 \mathrm{~nm} / \mathrm{min}$. $\mathrm{N}=291 \mathrm{spots}$. WIN, $64.6 \pm 2.5 \mathrm{~nm} / \mathrm{min} . N=225$ spots. ${ }^{\star \star \star} p<0.0001$, statistical analysis was performed by unpaired two-tailed Student's $t$ test. Data in (c, $\mathbf{f}, \mathbf{h}$ ) are mean \pm s.e.m. ( $N=3$ biological replicates; 70-120 axonal regions were examined per condition). Source data are provided as a Source Data file.

$\mathrm{CB}_{1}$ agonist, WIN. Periodic $\mathrm{CB}_{1}$ hotspots were confined (Fig. $4 \mathrm{~d}$ ). $\mathrm{CB}_{1}$ displayed higher periodicity as reflected by the higher autocorrelation amplitude (Fig. 4d, e). Therefore, our results show that with WIN application, active $\mathrm{CB}_{1}$ moved around their original point to a lesser extent than nonactive $\mathrm{CB}_{1}$ (Fig. $4 \mathrm{e}$ ).

Next, we used fluorescence recovery after photobleaching (FRAP) experiments to examine the recovery rate of $\mathrm{CB}_{1}$ after photobleaching to further study the dynamics of $\mathrm{CB}_{1}$ in culture neurons. We transfected the neurons with $\mathrm{CB}_{1}-\mathrm{RFP}$. We then measured the half-time recovery rate of $\mathrm{CB}_{1}$. Our results showed that with WIN application the half-time recovery of $\mathrm{CB}_{1}$ was slower than in the control, while this effect was blocked with the application of $\mathrm{CB}_{1}$ antagonist (Supplementary Fig. 9a-c). The half-time recovery of $\mathrm{CB}_{1}$ was not affected by the application of agonists of other types of GPCRs, suggesting that the change was likely caused by $\mathrm{CB}_{1}$ activation (Supplementary Fig. 9c). Thus, our results show that $\mathrm{CB}_{1}$ displayed less dynamics with receptor activation.

$\mathrm{CB}_{1}$ signaling is related to cytoskeleton. To evaluate the dependence of $\mathrm{CB}_{1}$ periodic hotspots on the cytoskeleton, we treated cultures with either latrunculinB (latB) or cytochalasin D (cytoD) to disrupt cytoskeletal structure ${ }^{8,14}$. We found that following both latB and cytoD treatments, periodic hotspots of $\mathrm{CB}_{1}$ were no longer observed (Fig. 5a, b), suggesting that the cytoskeleton is important for maintaining the $\mathrm{CB}_{1}$ structure in neurons. Next, we tested whether the cytoskeleton affects $\mathrm{CB}_{1}$ intracellular downstream signaling. $\mathrm{CB}_{1}$ can activate both Akt and ERK1/2 1,22 . In primary neuron cells, pretreatment with latB resulted in decreased phosphorylation levels of both Akt and ERK1/2 in a dose-dependent manner (Supplementary Fig. 10a, b). Thus, these results suggest that the intracellular signaling of $\mathrm{CB}_{1}$ is dependent upon the MPS cytoskeleton (Fig. 5c-e).

\section{Discussion}

Visualization of GPCRs in native tissue is necessary for understanding the intracellular organization of these receptors in real physiology. With the recent development of super-resolution imaging methods, we now can observe the organization of GPCRs at the cellular and tissue levels ${ }^{12,11}$. It is also important to visualize the live dynamics of GPCRs in order to understand potential functional changes in physiological and pathological 
a
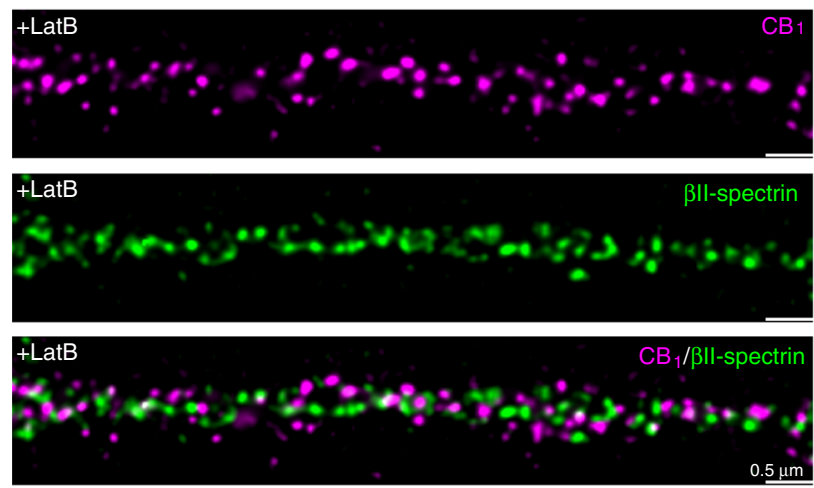

b
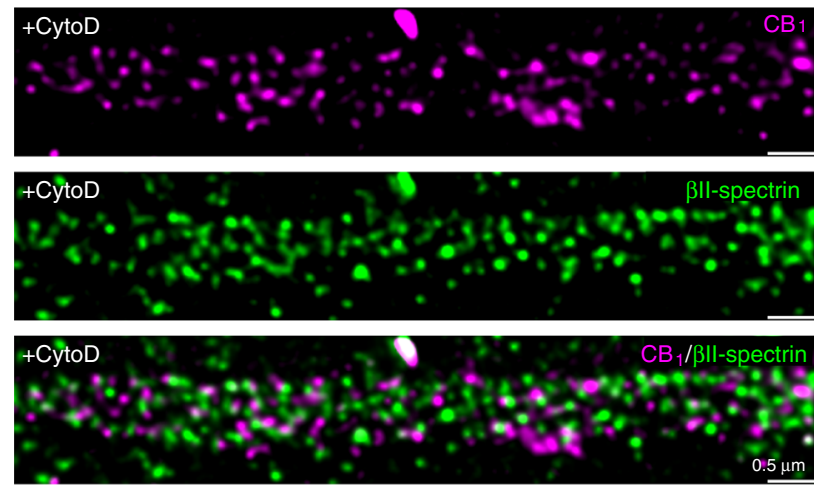

c
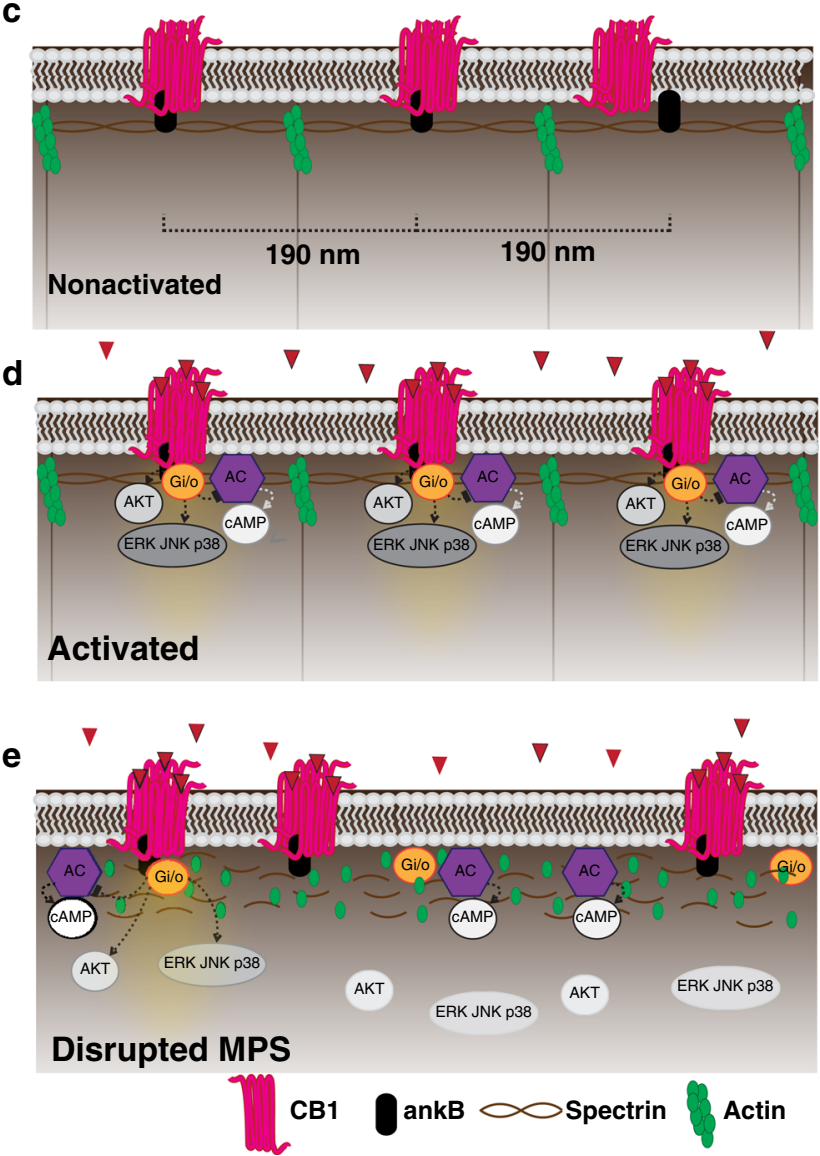

Fig. 5 Schematic illustration showing $\mathbf{C B}_{\mathbf{1}}$ forming dynamic peri-periodic hotspots to increase signaling efficiency. $\mathbf{a}$, $\mathbf{b}$ Two-color $S T E D$ images of $C B_{1}$ (magenta) and $\beta \|$-spectrin (green) in neurons treated with LatB (a) and CytoD (b). $N=3$ biological replicates. c Without ligand binding, MPS sets the range for $\mathrm{CB}_{1}$ distribution. $\mathbf{d}$ Upon ligand binding, active $\mathrm{CB}_{1}$ are recruited to the MPS and become more periodic, making downstream $A K T$ and ERK signaling more effective. e MPS degradation leads to less strong periodic clusters of $\mathrm{CB}_{1}$ and thus less downstream signaling.

conditions. Here, we used SIM to investigate the dynamics of $\mathrm{CB}_{1}$. Our findings with imaging probes likely reflect the dynamics of $\mathrm{CB}_{1}$ in neurons as both probes in live neurons displayed a spatial pattern similar to that of $\mathrm{CB}_{1}$ antibody in fixed neurons. Previous studies have shown that GPCRs form homodimers, heterodimers, or oligomers to affect their downstream signaling pathways ${ }^{23-26}$.

Uncovering the cellular structure of GPCRs has proven to be a challenging task, as several prior studies did not observe the semiperiodic organization of $\mathrm{CB}_{1}$ as we $\operatorname{did}^{12,11}$. An early study using STORM to characterize the $\mathrm{CB}_{1}$ structure in brain tissue did not observe any sign of organized $\mathrm{CB}_{1}$ pattern in axons. This could be due to the use of different antibodies, though most is likely because they did not focus on $\mathrm{CB}_{1}$ in the axonal shaft region, but instead focused on axonal boutons ${ }^{12}$. Intriguingly, Zhou et al. ${ }^{11}$ found that $\mathrm{CB}_{1}$ displays a nonperiodic structure without the application of WIN, an agonist of $\mathrm{CB}_{1}$, and becomes periodic upon the administration of WIN.

Our results using the same antibody used in Zhou's experiments showed both periodic and nonperiodic hotspots in both culture neurons and native brain tissue. We observed the periodicity of $\mathrm{CB}_{1}$ clusters in live neurons to avoid artifacts caused by fixation procedures. Further, we found that active $\mathrm{CB}_{1}$ may behave differently than native ones, as $\mathrm{CB}_{1}$ displayed less dynamic and more confined movements upon WIN application. Our molecular biology and imaging results suggest that the ICL3 may participate in the association between $\mathrm{CB}_{1}$ and MPS components, such as ankB and spectrin. In the presence of $\mathrm{WIN}, \mathrm{CB}_{1}$ can be activated and then some residues of ICL3 region are phosphorylated and contain more negative charges. By this way, more negative charged residues after receptor activation could attract and bind to the positive parts of the disordered domains of ankB and thus leading to a stronger connection between $\mathrm{CB}_{1}$ and ankB. This could explain the less movement of $\mathrm{CB}_{1}$ in the presence of WIN. The exact molecular mechanism underlying the distinct dynamics of $\mathrm{CB}_{1}$ is an important question and deserves further investigation.

Actually, Zhou's data implied that there was weak $\mathrm{CB}_{1}$ periodicity and colocalization with spectrin at $\sim 190 \mathrm{~nm}$ before agonist stimulation ${ }^{11}$. In the native state, we found that axons contained a semi-periodic pattern. Most synaptic sites did not exhibit periodic pattern, implying that the molecular mechanism to organize the synapses and $\mathrm{CB}_{1}$ complexes may be different. A small population of AISs had $\mathrm{CB}_{1}$ expression, while the others did not contain $\mathrm{CB}_{1}$ at all. This might be due to the fact that a small population of primary neurons in culture are inhibitory. The periodic distribution without application of WIN could be explained by the fact that constitutive released ligand, such as 2-AG, may activate $C_{1}$ to a certain extent and thus lead to the periodic pattern of $\mathrm{CB}_{1}$. However, in the presence of antagonist, $\mathrm{CB}_{1}$ still remained periodic in some regions of axons in primary culture neurons and its overall periodic pattern was not different. This suggests that the periodic organization of $\mathrm{CB}_{1}$ in native state might not be caused by constitutive released ligand in culture neurons, though we could not exclude this possibility in the brain tissue. 
In conclusion, we found that $\mathrm{CB}_{1}$ is distributed in axons as organized hotspots separated by approximately $190 \mathrm{~nm}$, in a similar spatial distribution to the MPS. Especially, $\mathrm{CB}_{1}$ tends to be more organized as periodic hotspots upon agonist application suggesting that active $\mathrm{CB}_{1}$ associate more strongly with the MPS. When a GPCR is activated by an agonist, it increases the kinetics of interaction with $G$ proteins and conducts downstream signal transduction within hotspots, which are usually confined to the cytoskeletal and clathrin-forming grids ${ }^{27}$. Our results suggest that active $C_{1}$ is clustered into hotspots where $G$ proteins or $\beta$ arrestin can easily collide with and form a relatively stable interaction with intracellular signaling components to increase signaling efficacy. Our results also indicate that $\mathrm{CB}_{1}$ are anchored to the MPS, likely by ankB, forming a fundamental structural unit that may be important for the proper function of neurons. These units likely form the structural basis for hotspots where signals transfer from extracellular to intracellular compartments. Our observation of periodic hotspots of $\mathrm{CB}_{1}$ along axon shafts, and the role of the cytoskeleton in $\mathrm{CB}_{1}$ 's intracellular signaling, indicate a horizon for the study of structural-functional interaction in neurons.

\section{Methods}

Animals. C57BL/6 mice, Sprague-Dawley rat, $\mathrm{CB}_{1}$ knockout mice (Biocytogen) and Kunming (KM) mice (for knockout mice generation) were used in this study. Male $\mathrm{C} 57 \mathrm{BL} / 6$ mice and $\mathrm{CB}_{1}$ knockout mice at 8-12 weeks were used. Animals were housed under a $12 \mathrm{~h}$ light/dark cycle at a room temperature of $22 \pm 1^{\circ} \mathrm{C}$ with $45 \%$ humidity, given ad libitum access to food and water. All experimental procedures were approved by the Institutional Animal Care and Use Committee of ShanghaiTech University, China.

Generation of Cnr1 knockout mouse. For Cnrl gene targeting, two sgRNAs were designed to target either the upstream or downstream region of its coding sequence by the CRISPR design tool (http://crispr.mit.edu) and screened for on-target activity using UCA ${ }^{\mathrm{TM}}$ (Universal CRISPR Activity Assay, Biocytogen). PCR amplification was performed to add the T7 promoter sequence to the Cas9 mRNA and sgRNAs DNA template and then the T7-Cas/sgRNA PCR products were gel purified. They were used as the template for in vitro transcription with the MEGAshortscript T7 kit (Cat. No. AM1354, Life Technologies). The Cas9 mRNA and sgRNAs products were purified with MEGAclear kit (Cat. No. AM1908, Life Technologies) and eluted with RNase-free water.

C57BL/6 female mice and KM mouse strains were used as embryo donors and pseudo-pregnant foster mothers, respectively. Superovulated female C57BL/6 mice (3-4 weeks old) were mated to C57BL/6 stud males, and fertilized embryos were collected from the ampullae. Different concentrations of Cas9 mRNA and sgRNAs were mixed and co-injected into the cytoplasm of one-cell stage fertilized eggs. After injection, surviving zygotes were transferred into oviducts of KM albino pseudo-pregnant females and allowed to develop to term.

Mutant mice were genotyped to ensure the deletion of target $\mathrm{CB}_{1}$ segment. To mitigate off-targets effects, mutant mice were crossed into $\mathrm{C} 57 \mathrm{Bl} / 6$ for five generations before being used for experimental purposes.

Primary culture of rat neurons. Sprague-Dawley rats of either sex at P0 were used for culturing rat hippocampal or cortex neurons. Brain tissues were isolated and digested with papain $\left(1 \mathrm{mg} / \mathrm{mL}\right.$; Sigma, P3125) at $37^{\circ} \mathrm{C}$ for $30 \mathrm{~min}$. The digested tissues were washed with Dulbecco's Modified Eagle Medium (DMEM) solution (Hyclone, SH30243.FS) three times and then transferred to culture medium containing Neurobasal medium (Thermo Fisher Scientific, 21103049) supplemented with $2 \%$ (vol/vol) B27 supplement (Thermo Fisher Scientific, 17504044) and 1\% (vol/vol) Glutamax (Thermo Fisher Scientific, 35050-061). The tissues were gently triturated until no chunks of tissue were left. Dissociated cells were then counted and plated onto poly-D-lysine-coated 12-mm coverslips (12$545-80$, Fisher Brand) or $29 \mathrm{~mm}$ Glass (\#1.5 cover glass) bottom dishes (D29-20$1.5-\mathrm{N}$, Cellvis). Neuronal cultures were maintained in the culture medium in a humidified atmosphere with $5 \% \mathrm{CO}_{2}$ at $37^{\circ} \mathrm{C}$. One-half of the medium was changed every 3 days to maintain neuron viability.

Fluorescence labeling of neurons. Cultured neurons were fixed with $4 \%$ (w/v) paraformaldehyde (PFA) in phosphate-buffered saline (PBS) for $15 \mathrm{~min}$ at 14 day in vitro (DIV 14). After a complete wash with PBS, the samples were then permeabilized and blocked in the blocking buffer ( $10 \% \mathrm{v} / \mathrm{v}$ donkey serum, $0.2 \% \mathrm{v} / \mathrm{v}$ Triton X-100 in PBS) for $1 \mathrm{~h}$ at room temperature, and subsequently stained with one or two primary antibodies in the incubation buffer ( $1 \%$ donkey serum, $0.1 \%$ Triton X-100 in PBS) overnight at $4^{\circ} \mathrm{C}$. The samples were washed in PBS and then stained with secondary antibodies as described above in the incubation solution for $1 \mathrm{~h}$ at room temperature. After incubation, samples were washed with PBS. Neuron samples were mounted with ProLong Gold (P36930, Life Technology) and imaged.

Perfusion and immunostaining of brain sections. Briefly, adult male C57BL/6 mice and $\mathrm{CB}_{1}$ knockout mice were anesthetized with sodium pentobarbital ( $40 \mathrm{mg} /$ $\mathrm{kg}$, i.p.) with no avoidance response to foot pinch. They were then perfused with normal saline (at $37^{\circ} \mathrm{C}$ ) and subsequently by ice-cold $4 \%$ PFA for fixation. Brains were post-fixed in $4 \%$ PFA for $4 \mathrm{~h}$, then dehydrated in $30 \%$ sucrose. Brains were frozen at $-80{ }^{\circ} \mathrm{C}$ and then sectioned at 20 - $\mu$ m-thick with the freezing microtome (Leica CM1950) for immunofluorescence labeling.

For the fluorescence immunostainings procedure, brain sections were rinsed in PBS, permeabilized, and then blocked with blocking solution (3\% w/v donkey serum and $0.3 \% \mathrm{v} / \mathrm{v}$ Triton X-100 in PBS) for $2 \mathrm{~h}$ at room temperature and then overnight at $4{ }^{\circ} \mathrm{C}$ with the primary antibody in $0.1 \%$ Triton $\mathrm{X}-100$ and $1 \%$ serum in PBS. After washing with PBS, sections were incubated with the secondary antibody in $0.1 \%$ Triton X-100 and $1 \%$ serum in PBS for $2 \mathrm{~h}$ at room temperature. After incubation, sections were washed with PBS. Sections were mounted with ProLong Gold mounting medium for following imaging.

STED imaging. Confocal and STED images were obtained at a Leica TCS SP8-3X gated STED system (Leica Microsystems) equipped with a pulsed white light laser (WLL, tunable from 470 to $670 \mathrm{~nm}$ ) for excitation, a $592 \mathrm{~nm} \mathrm{CW}$ laser (MPB Communications), a $660 \mathrm{~nm}$ CW laser (MPB Communications), and a $775 \mathrm{~nm}$ pulsed laser (Onefive) for depletion. The system includes a $\times 100$ objective lens (Leica, HC PL APO CS $2 \times 100 / 1.40$ oil), two HyD detectors, and the TCS SP8 timegated system. Each time before imaging, the depletion laser was co-aligned with the excitation laser. By STED effect, the lateral fluorescence was filtered out, leaving the remaining fluorescence in the center to be detected.

For single or dual color $2 \mathrm{D}$ imaging on the primary neuron culture, samples were excited by the WLL at $488 \mathrm{~nm}$ (Alexa Fluor 488-labeled $\beta I I-s p e c t r i n$ or ankB) and $561 \mathrm{~nm}$ (Alexa Fluor 555-labeled CB1) and depleted by 592 and $660 \mathrm{~nm}$ laser, respectively. The emission spectrums were set at $493-550 \mathrm{~nm}$ and $566-600 \mathrm{~nm}$ accordingly. Channels were recorded sequentially. Acquisition parameters were optimized as follows: logical size, $1024 \times 1024$ pixels; pixel size, $23 \mathrm{~nm}$; scan speed, $400 \mathrm{~Hz}$; scan direction, unidirectional; line average 4, no frame average; optical zoom factor 5, pinhole 1 airy disc; detector gain, 100\%; HyD time gating, 0.3-6 ns for confocal and 1-6 ns for STED; excitation power 10-30 pW; STED power,

$11-17 \mathrm{~mW}$ for $592 \mathrm{~nm}$ and $5-8 \mathrm{~mW}$ for $660 \mathrm{~nm}$.

For single-color imaging on fixed brain slices, CB1 was labeled with Alexa Fluor 647, excited by the WLL at $633 \mathrm{~nm}$ and depleted by $775 \mathrm{~nm}$ laser. The emission spectrum was set at $657-700 \mathrm{~nm}$. In order to reduce the adverse effect of aberration and scatter caused by the thickness of tissue, only those processes in close proximity ( $<1 \mu \mathrm{m}$ in $z$ axis) and parallel to cover glasses were chosen to acquire $2 \mathrm{D}$ images. The HyD time gating was set at $0.3-6 \mathrm{~ns}$ for confocal and $0.5-6 \mathrm{~ns}$ for STED, and the power of $633 \mathrm{~nm}$ laser and $775 \mathrm{~nm}$ laser was $20-30 \mathrm{pW}$ and 7-8 $\mathrm{mW}$, respectively. Other acquiring parameters were similar to the above.

Deconvolution of STED images was performed by Huygens software (Scientific Volume Imaging) with the Huygens classical maximum likelihood estimation (CMLE) deconvolution algorithm and theoretical Point Spread Functions. The deconvolution was performed by using Deconvolution Wizard. Deconvolution parameters were set to match the refractive index (RI) of the mounting media (RI of ProLong Gold polymerized until complete dry was 1.47) and the RI of the objective oil (1.518). Detailed deconvolution parameters were as follows, background, automatic estimation; estimate mode, lowest; area radius, 0.7; deconvolution algorithm, CMLE; maximum iteration, 40; signal-to-noise ratio, 7-10; quality threshold, 0.001; iteration mode, optimized; PSFs per brick, one PSF; brick layout, auto.

To measure the spatial resolution of the STED imaging microscope, $20 \mathrm{~nm}$ fluorescent microspheres (F8887, Thermo Fisher Scientific) were used to measure the performance of microscope. Beads were diluted 100 -fold with $\mathrm{ddH} 2 \mathrm{O}$ and then sonicated in order to disperse bead aggregates. $1 \mu \mathrm{L}$ beads were fully spread on a 0.17 -um-thick and PLL-coated coverslip. After about ten minutes with those beads dried, coverslips were mounted on the slide with a thin layer of mounting media (ProLong Gold). The slide was kept at room temperature until the ProLong Gold was fully dried. The microspheres were excited by a $561 \mathrm{~nm}$ laser and depleted by a $660 \mathrm{~nm}$ laser. The acquiring parameters were optimized as follows: logical size, $1024 \times 1024$ pixels; pixel size, $11 \mathrm{~nm}$; scan speed, $400 \mathrm{~Hz}$; scan direction, unidirectional; line average 4, no frame average; optical zoom factor 10; pinhole 1 airy disc; detector gain, 100\%; HyD time gating, 0.3-6 ns for confocal and 1-6 ns for STED; excitation power, $30-40 \mathrm{pW}$; STED power, $15 \mathrm{~mW}$. Intensity profiles across the center of beads were plotted in the FIJI software, and the FWHM (Full width at half maximum) was calculated by fitting with a Gaussian function ${ }^{28}$.

Live SIM imaging the structure of $\mathbf{C B}_{\mathbf{1}}$ in neurons. SIM images were obtained with the GE DeltaVision OMX microscope, equipped with a 568-nm laser (Coherent), a $\times 60$ objective lens (Olympus, PL Apo $\mathrm{N} \times 60 / 1.42$ oil), and the scientific CMOS camera (Acquisition pixel size, $82 \mathrm{~nm}$ at $\times 60$ objective; PCO edge). $3 \mathrm{D}$-SIM mode was acquired with a fixed $512 \times 512$ pixel size and an optical section 
space of $0.125 \mu \mathrm{m}$. Fifteen images were taken with three illumination angles and five phase shifts for each $Z$-section. Images were taken in fast $286 \mathrm{MHz}$ mode.

Primary neurons were transiently transfected using lipofectamine 2000 (Invitrogen) at DIV 9-12 (2 $\mu \mathrm{g}$ plasmid DNA/20 mm dish). Briefly, neurons were mixed with transfection complexes containing $\mathrm{CB}_{1}$-RFP plasmid DNA and lipofectamine 2000 at a ratio of 1:2 in MEM medium (Thermo Fisher Scientific, 11090081) for $2 \mathrm{~h}$, then subsequently incubated with the culture medium. One day after transfection, neurons expressed $\mathrm{CB}_{1}-\mathrm{RFP}$ were imaged under the live $3 \mathrm{D}$-SIM mode. The cells were incubated with Neurobasal medium (without phenol red, Thermo Fisher Scientific, 12348017) at $37^{\circ} \mathrm{C}$ and supplied with $5 \% \mathrm{CO}_{2}$ mixed with $95 \%$ air during the imaging process. To obtain images with minimized spherical aberration and optimized illumination contrast, immersion oil with different refractive index (RI) were systematically optimized for each individual sample until a symmetrical point spread function was achieved. Usually the oil with RI 1.520 was chosen. To reduced phototoxicity, the illumination laser intensity and the exposure time were carefully adjusted to a minimum value ( $10 \%$ of maximum laser intensity; exposure time, $6-10 \mathrm{~ms}$ ) and only $1 \mu \mathrm{m}$ in depth (eight optical sections) were acquired. The time series of 3D-SIM images were acquired six frames with 1 or $3 \mathrm{~min}$ acquisition interval. An ultimate focus system was used to maintain the sample $Z$ position, regardless of the mechanical and thermal changes during the acquisition.

The raw SIM images were reconstructed by the OMX SI reconstruction tool available in softWoRx (GE). Channel-specific OTF files, channel-specific K0 angles, Wiener filter constant (0.001), and bias offset (65) were used during the reconstruction. Widefield images were generated by averaging phase steps from SIM raw images.

To detect the SIM microscope resolution, the bead samples were made similarly to those for STED, but use Vectashield (H-1000, Vectorlabs) as mounting medium instead of Prolong Gold. Slides were sealed with nail polish and imaged. The imaging procedure was similar to the live SIM imaging. FWHM was determined with the same method to STED.

After SIM reconstruction, the images were processed in the FIJI software. The rigid image drift during acquisition was corrected with "Correct 3D drift" plugin ${ }^{29}$. Images were maximum-intensity projected and the linearly contrast was adjusted using Fiji software (National Institutes of Health). For the analysis and display, only those examples that could be tracked and did not bleach more than $20 \%$ for consecutive three time frames were selected. Fiji TrackMate was then used to analyze the dynamics of $\mathrm{CB}_{1}$ hotspots in these images ${ }^{30}$. In TrackMate, the difference of Gaussian (DoG) detector was set with an estimated spot diameter of $80 \mathrm{~nm}$ and an appropriate fluorescence intensity as the threshold to detect all the individual $\mathrm{CB}_{1}$ hotspots. The Simple Linear Assignment Problem (LAP) tracker with a linking maximum distance of $200 \mathrm{~nm}$, a gap-closing maximum distance of $200 \mathrm{~nm}$, and a gap-closing maximum frame gap of 2 was used for tracking cells through the time course images.

In situ PLAs. The protocol for PLA can be found on Duolink in situ PLA detection Kit (Sigma) following the instructions of the supplier with slight modifications ${ }^{31}$. Neurons at DIV 14-18 were fixed and incubated with primary antibodies overnight as described in the section of immunofluorescence staining. On the next day neurons were washed $3 \times$ with Phosphate-Buffered Saline, 0.1\% Tween ${ }^{\circledR} 20$ Detergent (PBST). All remaining steps were carried out in a $37^{\circ} \mathrm{C}$ humidified chamber. To detect $\mathrm{CB}_{1}-$ cytoskeleton protein interaction, a mixture of equal amounts of anti- $\mathrm{CB}_{1}$ antibody directly linked to a plus PLA probe and cytoskeleton protein antibody (like ankyrin B or $\beta$ II-spectrin) directly linked to a minus PLA probe was used. Neurons were incubated with a pair of PLA probes diluted 1:10/ each in $1 \times$ blocking solution for $1 \mathrm{~h}$, before washing $3 \times$ at 10 min each with $1 \times$ Washing Buffer-A. Ligase was diluted 1:40 into $1 \times$ Ligation buffer and added to neurons for $0.5 \mathrm{~h}$ ligation, followed by $3 \times 5 \mathrm{~min}$ washes with $1 \times$ Washing Buffer-A. Polymerase was then applied 1:80 to neurons in $1 \times$ amplification stock for $2.5 \mathrm{~h}$. Neurons were washed $2 \times 10$ min with $1 \times$ Washing Buffer-B, $1 \times 10$ min with $0.01 \times$ Washing Buffer-B, and $1 \times 10$ min with PBST. Neurons were fixed again by $4 \%$ PFA for 10 min to better retain the PLA signals before incubated with axonal primary antibody like MAP2 or Tuj1 at room temperature for $2 \mathrm{~h}$. Then neurons incubated with fluorescent secondary antibody for $1 \mathrm{~h}$ at room temperature before mounting with Mounting Medium with 4',6-Diamidino-2-Phenylindole, Dihydrochloride (DAPI). The samples were observed in a Nikon confocal microscope equipped with an apochromatic $\times 60$ oil-immersion objective (N.A. 1.4), and a 405 , a 488 and a $561 \mathrm{~nm}$ laser line. For each field of view, a stack of three channels (one per staining) and $10-15 Z$ stacks with a step size of $1 \mu \mathrm{m}$ were acquired. Images were opened and processed with Fiji software. The ratio $r$ (number of red spots/ number of cells containing spots) was determined considering a total of 300-600 cells from 6 to 10 different fields.

Fluorescence recovery after photobleaching. FRAP experiments on the culture neuron transfected with $\mathrm{CB}_{1}$-RFP were also performed on the GE DeltaVision OMX microscope with a $\times 60,1.47 \mathrm{NA}$ objective. Culture neurons were transfected with $\mathrm{CB}_{1}-\mathrm{RFP} 1$ day before imaging. Prior to the experiment, the medium was half changed to Neurobasal without phenol red. The cells were maintained at room temperature with $5 \% \mathrm{CO}_{2}$ mixed with $95 \%$ air supplied during the whole imaging process. Each FRAP image was taken with a fixed laser intensity and exposure time
(7-10 ms per frame) and 1-s interval. No images were acquired during the bleach period. Putative axonal regions transfected with $\mathrm{CB}_{1}-\mathrm{RFP}$ were chosen and an image stack of $1-2 \mu \mathrm{m}$ with $0.4-\mu \mathrm{m}$ interval in in $Z$ axis were acquired. Ultimate focus system was also used to maintain the stability of the microscope. Six pre-bleach images were taken followed by bleaching a $1-\mu \mathrm{m}$-diameter circle region for $0.1 \mathrm{~ms}$ with a strong laser intensity. Immediately after bleach, post-bleach images were taken until the recovery reached a complete steady state. Images were maximum projected and processed in Fiji. Briefly, FRAP data were quantified using Fiji software. During image processing, the background level was subtracted from each frame to quantify the recovery of fluorescence. Average fluorescence intensity of the bleached area was corrected for additional bleaching during regular imaging and was normalized to prebleach intensity. The image fluorescence over time was normalized to initial fluorescence (average fluorescence value from the 5-s imaging period immediately before photobleaching, defined as 100\%) and immediately after bleaching ( $0 \%$ ). Analysis of the recovery fitting curves and the half-time recovery were carried out with the Fiji macro (http://imagej.net/Analyze_FRAP_movies_with_a_Jython_script) ${ }^{32}$.

Western blot. Primary neurons were either treated or not treated with the indicated ligands for the times noted, rinsed with ice-cold PBS, and lysed by the addition of $100 \mu \mathrm{L}$ of ice-cold RIPA lysis buffer (Beyotime) with protease inhibitor cocktail (Roche). Cellular debris was removed by centrifugation at $13,000 \times g$ for 15 min at $4{ }^{\circ} \mathrm{C}$, and the amount of protein was quantified by BCA protein assay kit (Pierce). Protein samples were separated on 10\% SDS-PAGE gels (Bio-Rad) and transferred onto PVDF (polyvinylidene fluoride) membranes (Millipore, Billerica, MA, USA). The membranes were blocked with $5 \%$ non-fat dry milk for $2 \mathrm{~h}$ at RT and incubated overnight at $4{ }^{\circ} \mathrm{C}$ with rabbit anti-phospho-ERK1/2 (1:2000, Cell Signaling), anti-phospho-Ser473-Akt (1:2000, Cell Signaling), anti-ERK1/2 (1:2000, Cell Signaling), and anti-AKT (1:2000, Cell Signaling) antibodies. The membranes were then incubated with HRP-conjugated secondary antibody (1:1000; Pierce) for $2 \mathrm{~h}$ at room temperature. Signals were visualized using enhanced chemiluminescence (ECL, Pierce, Rockford, IL), and captured by the ChemiDoc XRS system (Bio-Rad Laboratories, CA). Phosphorylated ERK1/2 or phosphorylated Akt levels were normalized for differences in loading using protein band intensities for total ERK1/2 or AKT

Immunoprecipitation. Following tetracycline induction, $\mathrm{CB}_{1}-\mathrm{CHO}$ cells were washed with ice-cold PBS and suspended in immunoprecipitation (IP) buffer containing (in $\mathrm{mM}$ ), 50 Tris- $\mathrm{HCl}, 120 \mathrm{NaCl}, 0.5 \%$ Nonidet P- 40 and protease cocktail $(\mathrm{pH}=7.5)$. The lysate was sonicated, centrifuged at $13,000 \times g$ for $20 \mathrm{~min}$ at $4{ }^{\circ} \mathrm{C}$, and the resulting supernatant was incubated with the rabbit anti-CB ${ }_{1}$ (CST, 93815) antibody for $20 \mathrm{~min}$ at $4^{\circ} \mathrm{C}$. Immuno-complex was incubated with Protein A-Magnetic beads overnight on a rotating wheel at $4{ }^{\circ} \mathrm{C}$ from the precleared supernatant with anti- $\mathrm{CB}_{1}$ antibody covalently coupled to Protein G-Magnetic beads. The pellet was then washed 5 times in wash buffer containing (in $\mathrm{mM}$ ), 20 Tris- $\mathrm{HCl}, 100 \mathrm{NaCl}, 1$ Ethylenediaminetetraacetic acid (EDTA), 0.5\% Nonidet P$40(\mathrm{pH}=8.0)$. The beads were to wash away any proteins nonspecifically bound to the beads. The immunoprecipitates were mixed with the loading buffer and resolved by SDS-PAGE. Western blots were performed with relevant antibodies. Rabbit IgG was used as a negative control.

\section{Mass spectra in brain tissues}

Mouse brain tissue preparation. Preparation of mouse brain membrane fractions was performed according to a previous study ${ }^{33}$. Briefly, six brain regions (olfactory bulb, cerebral cortex, cerebellum, hippocampus, midbrain, and spinal cord) were obtained from 9-week-old C57BL/6 wild-type male mice. Brain regions from three mice were pooled and homogenized in the buffer of $300 \mathrm{mM}$ sucrose, $0.5 \%$ bovine serum albumin (BSA), $100 \mathrm{mM}$ EDTA, $30 \mathrm{mM}$ Tris/HCl, $\mathrm{pH} 7.4$ with protease inhibitor (Roche). Crude membrane fractions were isolated from the homogenate by ultra-centrifugation at $160,000 \times \mathrm{g}$ at $4{ }^{\circ} \mathrm{C}$ for $1 \mathrm{~h}$. The membrane pellet was solubilized in $4 \%$ SDS and $100 \mathrm{mM}$ dithiothreitol (DTT) in $100 \mathrm{mM} \mathrm{Tris} / \mathrm{HCl}, \mathrm{pH}$ 7.6, denatured and reduced at $95^{\circ} \mathrm{C}$ for $3 \mathrm{~min}$. Protein concentration was determined using the BCA assay. For each brain region, protein sample preparation was conducted in duplicate.

Protein digestion. The SDS-assisted digestion of membrane proteins was performed according to methods described previously ${ }^{34}$. Briefly, $50 \mu \mathrm{g}$ of protein was diluted in $8 \mathrm{M}$ urea, $50 \mathrm{mM} \mathrm{NH}_{4} \mathrm{HCO}_{3}$, and exchanged to the same buffer using the 30 KDa MWCO centrifugal filter unit (Satorious, Germany) by centrifugation at $13,000 \times g$ for $20 \mathrm{~min}$. The following centrifugation steps were performed under the same conditions. Subsequently, $100 \mu \mathrm{L}$ of $50 \mathrm{mM}$ iodoacetamide in $8 \mathrm{M}$ urea, $50 \mathrm{mM} \mathrm{NH}_{4} \mathrm{HCO}_{3}$ was added and incubated at room temperature in darkness for $30 \mathrm{~min}$, then followed by centrifugation. The concentrate was diluted with $200 \mu \mathrm{L}$ $50 \mathrm{mM} \mathrm{NH}_{4} \mathrm{HCO}_{3}$ and centrifuged again, this step was repeated twice. Proteins were digested with trypsin (Promega, Madison, USA) at an enzyme-to-protein ratio of $1: 100(\mathrm{w} / \mathrm{w})$ at $37^{\circ} \mathrm{C}$ for $3 \mathrm{~h}$, followed by the addition of trypsin at $1: 50(\mathrm{w} /$ w) and incubation at $37^{\circ} \mathrm{C}$ overnight. After acidification, the protein digest was desalted with C18-SepPak columns (Waters, Milford, USA) and lyophilized under vacuum. 
NanoLC-MS/MS analysis. The nanoLC-MS/MS analysis was conducted on an EASY-nLC 1000 connected to Orbitrap Fusion mass spectrometer (Thermo Fisher Scientific, USA) with a nano-electrospray ionization source. The eluted peptides were separated on an analytical column $(200 \mathrm{~mm} \times 75 \mu \mathrm{m})$ in-house packed with C18-AQ $3 \mu \mathrm{m} \mathrm{C18} \mathrm{resin} \mathrm{(Dr.} \mathrm{Maisch,} \mathrm{GmbH,} \mathrm{Germany)} \mathrm{over} \mathrm{a} \mathrm{130-min} \mathrm{gradient}$ at flow rate of $300 \mathrm{~nL} / \mathrm{min}$. For a pooled sample from all six brain region protein digests, a data-dependent (DDA) acquisition method was first employed with the following parameters, resolution of 60,000 used for survey scans; the mass range pf $300-1700 \mathrm{~m} / \mathrm{z}$; an AGC target value of 4E5; and maximum ion injection time of $50 \mathrm{~ms}$. Up to 12 dynamically chosen and most abundant precursor ions were fragmented. The MS/MS scans were acquired at an Orbitrap resolution of 30,000 (AGC target value 1E5, maximum ion injection time $50 \mathrm{~ms}$ ).

In order to achieve the accurate quantification of selected proteins in the membrane fractions, we developed parallel-reaction-monitoring (PRM) MS assays for all proteins of our interest based on the protein identification results from the DDA experiment ${ }^{35}$. The PRM acquisition method started with a full scan event followed by targeted MS/MS for specific peptides from the proteins of interest. Major parameters for the MS/MS event in Orbitrap were a resolution of 30,000; an AGC target value of 2E5; and maximum injection time of $100 \mathrm{~ms}$. Peptide precursor ions in different brain region protein digests were monitored in the PRM assay by scheduling an inclusion list of each precursor with an isolation window of $1.6 \mathrm{~m} / \mathrm{z}$ and retention time shift of $2 \mathrm{~min}$.

MS data processing. Mass spectra from the DDA experiment were processed using Proteome Discoverer 2.1 against the Uniprot mouse sequence database. The search parameter included cysteine carbamidomethylation as a fixed modification and oxidation of methionine as variable modification. Precursor ion mass tolerance was set to $10 \mathrm{ppm}$ and fragment ion tolerance was $0.02 \mathrm{Da}$. Trypsin was set as the specific enzyme and two missed cleavages were allowed. The required false discovery rate (FDR) was set to $1 \%$ at the peptide and protein level.

For PRM data analysis, the Skyline software (v3.7.0) was used for targeted peptide quantification with settings specified by the software instruction ${ }^{36}$. Only $b$ or $y$-product ions with $m / z$ values greater than the precursor were selected to quantify the peptides. All the transitions were validated using the mProphet algorithm in Skyline advanced peak picking model that restricts the FDR to $<1 \%$. The peptide quantification was derived from the sum of the peak areas of 3 -6 product ions for selected peptides. Protein intensity was based on the summed MS responses of one to three unique peptides of the corresponding protein.

Quantification and statistical analysis. All image data were first processed with Fiji software (National Institutes of Health). Images were resized with the Bicubic interpolation and the brightness and contrast were linearly adjusted. To quantitatively analyze the distribution properties of $\mathrm{CB}_{1}$, segmented lines across the structures were drawn, the intensity profiles along the lines were measured and further analyzed in Matlab (MathWorks, Inc.). Individual fluorescence peaks were found and the distance between the neighboring peaks were calculated and pooled together. For distribution pattern analysis, autocorrelation and crosscorrelation were performed on the fluorescence intensity profiles. The correlation curve was pooled and averaged from many randomly selected lines. All intensity, distance and correlation data were plotted using Graphpad prism (Graphpad Software, Inc.), and all figure layouts were composed in Illustrator (Adobe Systems, Inc.).

Results were reported as mean \pm s.e.m. Statistical analysis of the data was performed using a Student's $t$ test, one-way ANOVA. Statistical significance was set at $p<0.05$.

\section{Data availability}

All data are available upon reasonable request. Source data are provided with this paper.

Received: 21 January 2020; Accepted: 15 October 2020;

Published online: 11 November 2020

\section{References}

1. Herkenham, M. et al. Cannabinoid receptor localization in brain. Proc. Natl Acad. Sci. USA 87, 1932-1936 (1990).

2. Agarwal, N. et al. Cannabinoids mediate analgesia largely via peripheral type 1 cannabinoid receptors in nociceptors. Nat. Neurosci. 10, 870-879 (2007).

3. Bellocchio, L. et al. Bimodal control of stimulated food intake by the endocannabinoid system. Nat. Neurosci. 13, 281-283 (2010).

4. Monory, K. et al. The endocannabinoid system controls key epileptogenic circuits in the hippocampus. Neuron 51, 455-466 (2006).

5. Ruehle, S. et al. Cannabinoid CB1 receptor in dorsal telencephalic glutamatergic neurons, distinctive sufficiency for hippocampus-dependent and amygdala-dependent synaptic and behavioral functions. J. Neurosci. 33, 10264-10277 (2013)

6. Hua, T. et al. Crystal structure of the human cannabinoid receptor CB1. Cell 167, 750-762.e714 (2016).

7. Hua, T. et al. Crystal structures of agonist-bound human cannabinoid receptor CB1. Nature 547, 468-471 (2017).

8. Xu, K., Zhong, G. S. \& Zhuang, X. W. Actin, spectrin, and associated proteins form a periodic cytoskeletal structure in axons. Science 339, 452-456 (2013).

9. Liu, Y. et al. Critical role of spectrin in hearing development and deafness. Sci. Adv. 5, eaav7803 (2019).

10. Reddy-Alla, S. et al. Stable positioning of Unc13 restricts synaptic vesicle fusion to defined release sites to promote synchronous neurotransmission. Neuron 95, 1350-1364.e1312 (2017).

11. Zhou, R., Han, B., Xia, C. \& Zhuang, X. Membrane-associated periodic skeleton is a signaling platform for RTK transactivation in neurons. Science 365, 929-934 (2019).

12. Dudok, B. et al. Cell-specific STORM super-resolution imaging reveals nanoscale organization of cannabinoid signaling. Nat. Neurosci. 18, 75-86 (2015).

13. Hell, S. W. Microscopy and its focal switch. Nat. methods 6, 24-32 (2009).

14. Zhong, G. S. et al. Developmental mechanism of the periodic membrane skeleton in axons. Elife 3, e04581 (2014).

15. Heintzmann, R. \& Huser, T. Super-resolution structured illumination microscopy. Chem. Rev. 117, 13890-13908 (2017).

16. Sanchez-Blazquez, P., Rodriguez-Munoz, M., Vicente-Sanchez, A. \& Garzon, J. Cannabinoid receptors couple to NMDA receptors to reduce the production of NO and the mobilization of zinc induced by glutamate. Antioxid. Redox Signal. 19, 1766-1782 (2013).

17. Polissidis, A. et al. The cannabinoid CB1 receptor biphasically modulates motor activity and regulates dopamine and glutamate release region dependently. Int. J. Neuropsychoph. 16, 393-403 (2013).

18. Bennett, V. \& Baines, A. J. Spectrin and ankyrin-based pathways, metazoan inventions for integrating cells into tissues. Physiol. Rev. 81, 1353-1392 (2001).

19. Schallmeiner, E. et al. Sensitive protein detection via triple-binder proximity ligation assays. Nat. Methods 4, 135-137 (2007).

20. Gardner, K. \& Bennett, V. Modulation of spectrin-actin assembly by erythrocyte adducin. Nature 328, 359-362 (1987).

21. Blazquez, $\mathrm{C}$. et al. The $\mathrm{CB}(1)$ cannabinoid receptor signals striatal neuroprotection via a PI3K/Akt/mTORC1/BDNF pathway. Cell Death Differ. 22, 1618-1629 (2015).

22. Franklin, J. M. \& Carrasco, G. A. Cannabinoid receptor agonists upregulate and enhance serotonin $2 \mathrm{~A}(5-\mathrm{HT}(2 \mathrm{~A}))$ receptor activity via ERK1/2 signaling. Synapse 67, 145-159 (2013).

23. Turu, G. \& Hunyady, L. Signal transduction of the CB1 cannabinoid receptor. J. Mol. Endocrinol. 44, 75-85 (2010).

24. Rozenfeld, R. et al. AT1R-CB(1)R heteromerization reveals a new mechanism for the pathogenic properties of angiotensin II. EMBO J. 30, 2350-2363 (2011).

25. Wickert, M. et al. The F238L point mutation in the cannabinoid type 1 receptor enhances basal endocytosis via lipid rafts. Front. Mol. Neurosci. 11, 230 (2018).

26. Perreault, M. L., Hasbi, A., O’Dowd, B. F. \& George, S. R. Heteromeric dopamine receptor signaling complexes, emerging neurobiology and disease relevance. Neuropsychopharmacology 39, 156-168 (2014).

27. Sungkaworn, T. et al. Single-molecule imaging reveals receptor-G protein interactions at cell surface hot spots. Nature 550, 543-547 (2017).

28. Nagerl, U. V. \& Bonhoeffer, T. Imaging living synapses at the nanoscale by STED microscopy. J. Neurosci. 30, 9341-9346 (2010).

29. Parslow, A. C., Cardona, A. \& Bryson-Richardson, R. J. Sample drift correction following 4D confocal time-lapse imaging. J. Vis. Exp. https://doi. org/10.3791/51086 (2014).

30. Tinevez, J. Y. et al. TrackMate, an open and extensible platform for singleparticle tracking. Methods 115, 80-90 (2017).

31. Tseng, W. C., Jenkins, P. M., Tanaka, M., Mooney, R. \& Bennett, V. Giant ankyrin-G stabilizes somatodendritic GABAergic synapses through opposing endocytosis of GABAA receptors. Proc. Natl Acad. Sci. USA 112, 1214-1219 (2015).

32. Woodruff, J. B. et al. The centrosome is a selective condensate that nucleates microtubules by concentrating tubulin. Cell 169, 1066-+ (2017).

33. Suski, J. M. et al. Isolation of plasma membrane-associated membranes from rat liver. Nat. Protoc. 9, 312-322 (2014).

34. Wisniewski, J. R., Zougman, A., Nagaraj, N. \& Mann, M. Universal sample preparation method for proteome analysis. Nat. Methods 6, 359-362 (2009).

35. Gallien, S., Kim, S. Y. \& Domon, B. Large-scale targeted proteomics using internal standard triggered-parallel reaction monitoring (IS-PRM). Mol. Cell. Proteom. 14, 1630-1644 (2015).

36. MacLean, B. et al. Skyline, an open source document editor for creating and analyzing targeted proteomics experiments. Bioinformatics 26, 966-968 (2010). 


\section{Acknowledgements}

We thank the Bioimaging Core Facilities of the iHuman Institute and the animal facility of National Center for Protein Science for their support. We thank Dr. Yulong Li from Peking University for providing the GPCR probes. This work was supported by the National Key Research and Development Program of China (2016YFC0905900 (G.Z.), 2017YFC 1001300 (G.Z.)), the National Natural Science Foundation of China (81970878 (G.Z.), 31871036 (T.W.), 31771130 (G.Z.), 81861128023 (G.Z.)), the 2015 Thousand Youth Talents Plan of China (G.Z.) and the Australian Research Council (Grant DE170100546 (T.W.)).

\section{Author contributions}

G.Z. initiated, managed and supervised the project. G.Z. and H.L. conceived and designed the experiments. H.L., J.Y., T.C., S.L., M.D., T.H., performed most of the experiments and data analysis. S.Z., Q.W., F.T., Y.Q., D.D.-G., G.T., Y.Z., W.S., T.W. and Z.-J.L. contributed to data analysis and interpretation. G.Z. wrote the manuscript with contributions from all of the authors.

\section{Competing interests}

The authors declare no competing interests.

\section{Additional information}

Supplementary information is available for this paper at https://doi.org/10.1038/s41467020-19510-5.
Correspondence and requests for materials should be addressed to G.Z.

Peer review information Nature Communications thanks the anonymous reviewers for their contributions to the peer review of this work.

Reprints and permission information is available at http://www.nature.com/reprints

Publisher's note Springer Nature remains neutral with regard to jurisdictional claims in published maps and institutional affiliations.

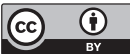

Open Access This article is licensed under a Creative Commons Attribution 4.0 International License, which permits use, sharing, adaptation, distribution and reproduction in any medium or format, as long as you give appropriate credit to the original author(s) and the source, provide a link to the Creative Commons license, and indicate if changes were made. The images or other third party material in this article are included in the article's Creative Commons license, unless indicated otherwise in a credit line to the material. If material is not included in the article's Creative Commons license and your intended use is not permitted by statutory regulation or exceeds the permitted use, you will need to obtain permission directly from the copyright holder. To view a copy of this license, visit http://creativecommons.org/licenses/by/4.0/.

(C) The Author(s) 2020 